\title{
Lithium Stabilizes the Mood of Bipolar Patients by Depolarizing the Neuronal Membrane Via Quantum Tunneling through the Sodium Channels
}

\author{
Abdallah Barjas Qaswal \\ School of Medicine, The University of Jordan, Amman, Jordan
}

\begin{abstract}
Objective: Lithium is used as first line in treating bipolar patients to stabilize their mood. However, the exact mechanism of lithium is not yet established. One of the proposed mechanisms is that lithium depolarizes the hyperpolarized neuronal membrane of bipolar patients bringing it back to the normal potential. On the other hand, the only way that lithium causes significant therapeutic depolarization is to have a membrane conductance that must be at least an order of magnitude higher than that for sodium but this is not achieved since both; lithium and sodium have the same conductance because the membrane channels are selective for them approximately by the same degree. So, this study aimed to explain how lithium could achieve higher conductance than sodium.

Methods: The idea of quantum tunneling through closed channels was used in a way to calculate the tunneling probability and the quantum conductance for lithium ions.

Results: It was found that lithium could achieve higher conductance than sodium because it has a smaller mass than sodium making lithium to have higher probability of tunneling and consequently higher conductance through channels and membrane.

Conclusion: Lithium tunneling model provides a reasonable explanation for the therapeutic depolarization effect of lithium. This model is experimentally testable to prove the tunneling effect of ions through the closed channels and to show the variations of quantum conductance between ions according to their mass.
\end{abstract}

KEY WORDS: Quantum tunneling; Sodium channel; Lithium; Bipolar disorder; Membrane potential.

\section{INTRODUCTION}

Lithium is the drug of choice in treating bipolar patients and also to prevent relapses [1-4]. However, the mechanism of action of lithium is not yet established [5,6] but one of the proposed mechanisms to explain the therapeutic effects of lithium is that it depolarizes the neuronal membrane of bipolar patients [7] since it was shown that these patients have hyperpolarized membrane and lithium ions bring the membrane potential back to the normal by depolarization [8].

Lithium ions are cations (positive ions) [9] that can de-

Received: July 2, 2019 / Revised: August 13, 2019

Accepted: August 19, 2019

Address for correspondence: Abdallah Barjas Qaswal

School of Medicine, The University of Jordan, Queen Rania Street, Amman 11942, Jordan

E-mail: qaswalabdullah@gmail.com

ORCID: https://orcid.org/0000-0001-5419-4793 polarize the neuronal membrane as sodium ions do in depolarization phase of action potential but this requires that the membrane conductance for lithium is at least an order of magnitude higher than the membrane conductance for sodium ions to induce a significant depolarization [7] which would be impossible since lithium and sodium ions have the same conductance through channels as they are selective for both cations by the same degree [10-12]. Accordingly, a different approach is needed to explain the significant depolarization effect of lithium ions; hence quantum mechanics might be able to explain that.

Quantum tunneling is quantum phenomenon in which small particles can pass through an energy barrier even though that their kinetic energy is less than the energy of that barrier [13]. Cations can tunnel through closed channels and induce depolarization as potassium ions do to cause well known clinical phenomenon called referred

(c) This is an Open-Access article distributed under the terms of the Creative Commons Attribution Non-Commercial License (http://creativecommons.org/licenses/by-nc/4.0) which permits unrestricted non-commercial use, distribution, and reproduction in any medium, provided the original work is properly cited. 
pain [14].

The aim of this study is to build a mathematical model of lithium ions tunneling through the closed sodium channels and to be used to explain how lithium ions could have a conductance higher than sodium conductance by more than an order of magnitude so that they can cause a significant depolarization in the neuronal membrane of bipolar patients.

\section{METHODS}

\section{Mathematical Modeling of Lithium Tunneling through Sodium Channels}

The therapeutic concentration of lithium is $0.6-1.2$ $\mathrm{mEq} / \mathrm{L}$ [15] with average value of $0.9 \mathrm{mEq} / \mathrm{L}$. Lithium ions do not equilibrate passively because there is active transport of lithium ions from inside the cell to the outside making the lithium cell to plasma ratio around 4 [16].

Using these numbers and applying them on Nernst equation [17], diffusion potential of lithium can be calculated:

$$
\begin{gathered}
V_{L i}(\text { millivolts })=-61 \times \log \frac{\left[\mathrm{Li}^{+}\right]_{i}}{\left[\mathrm{Li}^{+}\right]_{o}} \\
V_{L i}=-36.73 \mathrm{mV}
\end{gathered}
$$

This means to block net diffusion from inside the cell to the outside due to concentration gradient, voltage difference of $36.73 \mathrm{mV}$ against the direction of lithium diffusion is needed. Besides, in this case, the concentration difference between inside and outside is $2.7 \mathrm{mEq} / \mathrm{L}$ and that means for plasma concentration of $0.9 \mathrm{mEq} / \mathrm{L}$, a $12.24 \mathrm{mV}$ is needed to stop lithium diffusion to the inside and for intracellular concentration of $3.6 \mathrm{mEq} / \mathrm{L}$, a 48.97 $\mathrm{mV}$ is needed to stop Lithium diffusion to the outside.

Lithium ions coming from extracellular fluid (plasma) and passing through the sodium channels reaching the intracellular gate will have a kinetic energy $\left(K E_{o}\right)$ due to the voltage difference of neuronal membrane of $65 \mathrm{mV}$ and extracellular lithium ions concentration $(12.24 \mathrm{mV})$ calculated by the following equation:

$$
K E_{o}=V \times e
$$

Where $V$ is the voltage difference and in this case, $V=$
$65+12.24=77.24 \mathrm{mV}$ and $e$ is the electron charge.

By substituting the values in equation (2), $K E_{o}=1.24 \times$ $10^{-20} \%$

On the other hand, intracellular lithium ions have kinetic energy $\left(K E_{i}\right)$ due to intracellular lithium ions concentration $(48.97 \mathrm{mV})$ and it is calculated using equation (2) to be $K E_{i}=0.78 \times 10^{-20}$ \%

Voltage - gated sodium channels are sealed off by intracellular activation gate which is formed by crossing the S6 segments of the protein structure of channels preventing the permeation of sodium and lithium ions [18]. The energy needed to open the gate is $16.1 \mathrm{kcal} /$ mole [19] = $11.19 \times 10^{-20} \mathrm{~J}$. It is obvious that the kinetic energy of intracellular and extracellular energy is less than the energy needed to open the gate and consequently, a quantum tunnelling probability can be calculated.

Alpha helix in the protein structure of the sodium channels has helical shape and is composed of turns and each turn has around 3.6 residues [20] with a length of $5.4 \times 10^{-10}$ $m$ which means $1.5 \times 10^{-10} \mathrm{~m}$ for each residue [21]. With average tilt angle $21^{\circ}$ of alpha helix with the plane of neuronal membrane [22], the vertical length $L_{\text {residue }}$ which represents the length of energy barrier by one residue that lithium ions should tunnel through and is calculated as:

$$
L_{\text {residue }}=R \sin \theta
$$

Where (R) is the length of one residue and $\theta$ is the average tilt angle, then $L_{\text {residue }}=5.38 \times 10^{-11} \mathrm{~m}$.

The intracellular activation gate of sodium channels seals off the permeation of ions by 4 hydrophobic residues [23] and these 4 residues collectively represents the energy barrier for lithium tunnelling and that means the overall length $L=4 \times 5.38 \times 10^{-11}=21.52 \times 10^{-11} \mathrm{~m}$. As said before the energy to open the gate is $11.19 \times 10^{-20}$ J but this energy must be divided along the length of the barrier because this energy represents the overall energy to open the gate. Additionally, when dividing the barrier into $(n)$ parts, the length of the barrier will be divided into $\Delta L=L / n$, and the energy of each part $U=11.19 \times 10^{-20} / n$ ensuring that $U$ is still higher than kinetic energy $(K E)$ and the difference $(U-K E)$ is higher than the uncertainty in the kinetic energy $\triangle K E$ calculated from the equation of the uncertainty principle for Heisenberg as the following:

$$
\Delta P \times \Delta L \geq \hbar \text { then: }
$$




$$
\Delta K E \geq \frac{\hbar^{2} n^{2}}{2 m L^{2}}
$$

Where $\mathrm{m}$ is the mass of lithium ion and $\hbar$ is reduced Planck constant.

So, the barrier should be divided into (n) parts in a way that $U>K E$ and $(U>K E) \geq \frac{\hbar^{2} n^{2}}{2 m L^{2}}$. Regarding the extracellular lithium, the limit for (n) value for the first condition $(\mathrm{U}>\mathrm{KE})$ is $n<9.024$ which means any value equals or more than 9.024 is not valid, on the other hand if $n=8.9$ is substituted, $\Delta K E \geq 0.082 \times 10^{-20} \mathrm{~J}$ and $(U-$ $K E)=0.0173 \times 10^{-20} \mathrm{~J}$ which means the second condition $\left((U-K E) \geq \frac{\hbar^{2} n^{2}}{2 m L^{2}}\right)$ is not met, as a result $n=8.9$ is not valid.

The goal is to choose the largest valid value for $(n)$ that meets the previous two conditions, so as an estimation $n=8.5$ can be chosen.

When $n=8.5, U=1.32 \times 10^{-20} \mathrm{~J}, \Delta L=2.53 \times 10^{-11} \mathrm{~m}$ and $K E_{o}=1.24 \times 10^{-20} \mathrm{~J}$, the probability $\left(T_{p}\right)$ of tunneling through $\Delta L$ of the barrier can be calculated by the following equation:

$$
\begin{gathered}
T_{p}=e^{-2 \Delta L K} \\
\text { Where } K=\frac{\sqrt{2 m(U-K E)}}{\hbar}
\end{gathered}
$$

By substituting all the values of the variables in the equations (5) and (6), $T_{p}=0.126$ and since lithium ion must tunnel all the parts of the barrier, the overall probability $T_{t}=T_{p}^{8.5}=2.26 \times 10^{-8}$.

Applying the same calculations on intracellular lithium and by choosing approximately the most appropriate $(n)$ value to be $12, U=0.93 \times 10^{-20} \mathrm{~J}, \Delta L=1.79 \times 10^{-11} \mathrm{~m}$, $K E_{i}=0.78 \times 10^{-20} \mathrm{~J}$, consequently the tunneling probability $\left(T_{p}\right)$ through a part of the barrier $\Delta L$ equals 0.135 and the total probability to tunnel through the entire barrier will be $3.66 \times 10^{-11}$.

\section{RESULTS}

\section{Calculating the Quantum Membrane Conductance of Lithium}

Lithium ions can tunnel through the gate of sodium channels; as a result a tunneling electric current through the channels is produced. However, the neuronal membrane is not fully embedded by sodium channels and that means the probability of lithium ions to hit the sodium channels should be calculated by the following equation:

$$
T_{h}=A_{\text {pore }} \times D_{\text {channels }}
$$

$A_{\text {pore }}$ is the pore surface area of the sodium channels and it is calculated by this equation $A=\pi r^{2}$ where $r$ is the radius of the pore and it is on average $2 \times 10^{-10} m$ [17] and $D_{\text {channels }}$ is the density of voltage-gated channels in the neuronal membrane which is 12,000 channels $/ \mu m^{2}$ [24]. This finally will give a probability of lithium ions to hit these channels with a value of $1.51 \times 10^{-3}$. This means that when the tunnelling electric current is calculated, an overall probability $\left(T_{C}\right)$ of tunneling probability $\left(T_{t}\right)$ and probability of hitting the channels $\left(T_{h}\right)$ must be considered:

$$
T_{c}=T_{t} \times T_{h}
$$

For extracellular lithium, $T_{C}=3.4 \times 10^{-11}$ and for intracellular lithium, $T_{C}=5.53 \times 10^{-14}$

Tunneling electric current density $\left(\mathrm{amp} / \mathrm{m}^{2}\right)$ can be calculated [13] by:

$$
I=\frac{e^{2} V K}{4 \pi^{2} L \hbar} T_{C}
$$

Where $e$ is the electron charge, $V$ is the voltage difference across the gate of the channel, $K$ is given in equation (6). $T_{C}$ is given in equation (8), $L$ is the length of the entire barrier and $\hbar$ is reduced planck constant.

Furthermore, by substituting the equation (9) in the equation of conductance $(\mathrm{C}=\mathrm{I} / \mathrm{V})$ the sodium channel conductance for lithium ions due to quantum tunnelling (Quantum Conductance) is given by the following equation:

$$
C_{Q L i}=\frac{e^{2} K}{4 \pi^{2} L \hbar} T_{C}
$$

Quantum conductance for extracellular lithium $C_{Q L i(o)}=$ $4.0 \times 10^{4} \mathrm{mho} / \mathrm{m}^{2}$ and for intracellular lithium $C_{Q L i(i)}=$ $88.48 \mathrm{mho} / \mathrm{m}^{2}$. 
Finally, quantum conductance through the neuronal membrane must be calculated by the following equation:

$$
C_{M L i}=C_{Q L i} \times A_{\text {pore }} \times D_{\text {channels }}
$$

For extracellular lithium ions, $C_{M L i(o)}=6.03 \mathrm{mmho} / \mathrm{cm}^{2}$ and for intracellular lithium $C_{M L i(i)}=0.013 \mathrm{mmho} / \mathrm{cm}^{2}$.

\section{DISCUSSION}

Quantum conductance of extracellular lithium equals $6.03 \mathrm{mmho} / \mathrm{cm}^{2}$ which is 150 times higher than the conductance of sodium (see the values below). On the other hand, the quantum conductance of intracellular lithium equals $0.013 \mathrm{mmho} / \mathrm{cm}^{2}$ which is around 3 times less than the conductance of sodium ions. According to that, the neuronal membrane favors more extracellular lithium to get inside than intracellular lithium to get outside and that means a net result of depolarization effect occurs.

To show the depolarization effect mathematically, the resting membrane potential should be calculated before and after adding lithium using Goldman-Hodgkin-Katz equation [17]:

$$
V(m V)=-58 \times \log \frac{C_{K^{+}}\left[\mathrm{K}^{+}\right]_{i}+C_{\mathrm{Na}^{+}}\left[\mathrm{Na}^{+}\right]_{i}+C_{\mathrm{C}^{-}}\left[\mathrm{Cl}^{-}\right]_{o}}{C_{K^{+}}\left[\mathrm{K}^{+}\right]_{o}+C_{\mathrm{Na}^{+}}\left[\mathrm{Na}^{+}\right]_{o}+C_{a^{-}}\left[\mathrm{Cl}^{-}\right]_{i}}
$$

Where $V$ is the resting membrane potential, $C$ is the conductance (the permeability) for the ions, []$_{o}$ is extracellular concentration of ions, and []$_{i}$ is intracellular concentration of ions.

Substituting the following values for giant squid axons at $18^{\circ} \mathrm{C}[25,26]$ in equation (12):

$$
\begin{aligned}
& C_{K^{+}}: C_{\mathrm{Na}^{+}}: C_{\mathrm{Cl}^{-}}=1.00: 0.04: 0.45 \\
& {[\mathrm{~K}]_{i}:[\mathrm{Na}]_{i}:[\mathrm{Cl}]_{i}=400: 50: 40} \\
& {[\mathrm{~K}]_{o}:[\mathrm{Na}]_{o}:[\mathrm{Cl}]_{o}=20: 440: 560} \\
& \mathrm{~V}=-62 \mathrm{mV} \text { (before adding lithium) }
\end{aligned}
$$

By adding lithium to equation (12), it becomes:

$$
\begin{aligned}
& V(m V)= \\
& -58 \times \log \frac{C_{K^{+}}\left[K^{+}\right]_{i}+C_{\mathrm{Na}^{+}}\left[\mathrm{Na}^{+}\right]_{i}+C_{G^{-}}[\mathrm{Cl}]_{o}+C_{M L i^{+}(i)}\left[\mathrm{Li}^{+}\right]_{i}}{C_{K^{+}}\left[K^{+}\right]_{o}+C_{\mathrm{Na}^{+}}\left[\mathrm{Na}^{+}\right]_{o}+C_{Q^{-}}\left[\mathrm{Cl}^{-}\right]_{i}+C_{M i^{+}(o)}\left[\mathrm{Li}^{+}\right]_{o}}
\end{aligned}
$$

Extracellular lithium concentration to extracellular so- dium concentration ratio is approximately [7] $0.6 \times 10^{-2}$ and in case of giant squid axon, extracellular lithium concentration would be $2.64 \mathrm{mEq} / \mathrm{L}$ and consequently, intracellular lithium would be $10.56 \mathrm{mEq} / \mathrm{L}$ (as mentioned before that the ratio in between is 4). By substituting lithium concentrations and their permeabilities obtained in results section, $\mathrm{V}=-56 \mathrm{mV}$ (after adding lithium).

It is obvious that lithium can depolarize the neuronal membrane because it can achieve a conductance higher than that for sodium. Lithium has a mass less than that for sodium making the tunneling probability and consequently the quantum conductance higher because the tunneling probability is inversely related to the mass of the particle.

Lithium tunneling model provides a reasonable explanation for the therapeutic depolarization effect of lithium. This model is experimentally testable to prove the tunneling effect of ions through the closed channels and to show the variations of quantum conductance between ions according to their mass.

\section{- Conflicts of Interest}

No potential conflict of interest relevant to this article was reported.

\section{REFERENCES}

1. Licht RW. Lithium: still a major option in the management of bipolar disorder. CNS Neurosci Ther 2012;18:219-226.

2. Dinan TG. Lithium in bipolar mood disorder: evidence suggests that lithium should still be first choice for prophylactic treatment. BMJ 2002;324:989-990.

3. Müller-Oerlinghausen B, Berghöfer A, Bauer M. Bipolar disorder. Lancet 2002;359:241-247.

4. Bschor T. Lithium in the treatment of major depressive disorder. Drugs 2014;74:855-862.

5. Klein PS, Melton DA. A molecular mechanism for the effect of lithium on development. Proc Natl Acad Sci U S A 1996;93: 8455-8459.

6. Malhi GS, Tanious M, Das P, Coulston CM, Berk M. Potential mechanisms of action of lithium in bipolar disorder. Current understanding. CNS Drugs 2013;27:135-153.

7. Thiruvengadam A. Effect of lithium and sodium valproate ions on resting membrane potentials in neurons: an hypothesis. J Affect Disord 2001;65:95-99.

8. El-Mallakh RS, Li R, Worth CA, Peiper SC. Leukocyte transmembrane potential in bipolar illness. J Affect Disord 1996; 41:33-37.

9. Krebs RE. The history and use of our earth's chemical elements: a reference guide. Santa Barbara: Greenwood Publishing 
Group;2006.

10. Jakobsson E, Argüello-Miranda O, Chiu SW, Fazal Z, Kruczek J, Nunez-Corrales S, et al. Towards a unified understanding of lithium action in basic biology and its significance for applied biology. J Membr Biol 2017;250:587-604.

11. Shimura M, Yuan Y, Chang JT, Zhang S, Campochiaro PA, Zack DJ, et al. Expression and permeation properties of the $K$ (+) channel Kir7.1 in the retinal pigment epithelium. J Physiol 2001;531(Pt 2):329-346.

12. Chatelain FC, Gazzarrini S, Fujiwara Y, Arrigoni C, Domigan C, Ferrara G, et al. Selection of inhibitor-resistant viral potassium channels identifies a selectivity filter site that affects barium and amantadine block. PLoS One 2009;4:e7496.

13. Serway RA. Physics for scientists and engineers: with modern physics. Philadelphia:Saunders College Publishing;1992.

14. Qaswal AB. A theoretical study to explain the referred pain phenomenon and its characteristics via quantum tunneling of potassium lons through the channels of neuronal membrane. NeuroQuantol 2019;17:43-52.

15. Finley PR, Warner MD, Peabody CA. Clinical relevance of drug interactions with lithium. Clin Pharmacokinet 1995;29: 172-191.

16. Ehrlich BE, Diamond JM. Lithium, membranes, and manic-depressive illness. J Membr Biol 1980;52:187-200.

17. Hall JE. Guyton and hall textbook of medical physiology: enhanced E-book. London:Elsevier Health Sciences;2010.
18. Catterall WA. Structure and function of voltage-gated sodium channels at atomic resolution. Exp Physiol 2014;99:35-51.

19. Chowdhury S, Chanda B. Estimating the voltage-dependent free energy change of ion channels using the median voltage for activation. J Gen Physiol 2012;139:3-17.

20. Lodish H, Darnell JE, Berk A, Kaiser CA, Krieger M, Scott MP, et al. Molecular cell biology. New York:Macmillan;2008.

21. Moran LA, Horton RA, Scrimgeour G, Perry M, Rawn D. Principles of biochemistry. Boston:Pearson;2012.

22. Bowie JU. Helix packing in membrane proteins. J Mol Biol 1997;272:780-789.

23. Oelstrom K, Goldschen-Ohm MP, Holmgren M, Chanda B. Evolutionarily conserved intracellular gate of voltage-dependent sodium channels. Nat Commun 2014;5:3420.

24. Ritchie JM, Rogart RB. Density of sodium channels in mammalian myelinated nerve fibers and nature of the axonal membrane under the myelin sheath. Proc Natl Acad Sci U S A 1977;74:211-215.

25. Hodgkin AL, Huxley AF. A quantitative description of membrane current and its application to conduction and excitation in nerve. J Physiol 1952;117:500-544.

26. McCormick DA. Membrane potential and action potential. In: Byrne JH, Heidelberger $R$, Waxham MN, editors. From molecules to networks. Cambridge:Academic Press;2014. $p$. 351-376. 